\title{
Macro-element modelling of suction-embedded plate anchors for floating offshore structures
}

\author{
Anderson Peccin da Silva ${ }^{1, *}$, Andrea Diambra $^{1}$ and Dimitris Karamitros ${ }^{1}$ \\ ${ }^{1}$ Department of Civil Engineering, University of Bristol, Queens Building, BS8 1TR, Bristol, UK
}

\begin{abstract}
This work presents a new macro-element model to predict the behaviour of Suction Embedded Plate Anchors (SEPLAs) for floating offshore structures during keying and loading stages. Differently from previously published models for anchors, this new model is characterised by (i) a non-associated plastic potential with the aim of improving the prediction of anchor trajectory for the whole displacement domain and for a large range of padeye offsets; and (ii) by a strain-hardening rule enabling to predict the force and displacement mobilisation from the early stages of the keying process. The model was calibrated against LDFE analyses and compared with a broad set of LDFE and centrifuge tests results. The model proves capable of reproducing anchor rotation and displacement with good accuracy for a wide range of padeye offsets and distinct studies from the literature.
\end{abstract}

\section{Introduction}

In recent decades, several anchoring solutions have been developed for floating offshore structures, notably wind turbines and oil and gas platforms: drag anchors, suction caissons and plate anchors, among others. Following the different installation techniques that have been proposed and developed, plate anchors have recently become a quite popular technical solution. Dove et al. [1] and Wilde et al. [2] introduced the concept of Suction Embedded Plate Anchor (SEPLA), which consists in a rectangular thin fluke with a shank installed vertically in the seabed through pressure inside a suction caisson. The mooring line attached to the padeye (i.e. the loading point) is then tensioned, making the anchor to rotate from its initial vertical position to an inclined position, approximately normal to the load applied by the mooring line [1]. This process of rotation during the pulling process is called keying, during which the anchor experiences vertical motion, resulting in loss of embedment [3]. The major concern associated with SEPLAs (and offshore plate anchors in general) is the loss of embedment, which results in an unrecoverable loss of capacity [4-5].

Many studies on experimental and numerical analysis of plate anchors have been published. However, experimental set-ups for anchor keying and pull-out can be rather complex; and finite-element analysis can be very time-demanding due to the large deformations experienced by the soil. Macro-element models, on the other hand, can be a helpful way of reproducing the mechanical response of soil-foundation interaction problems at very low computational costs [6].

Cassidy et al. [7] proposed the only macro-element model capable of predicting the behaviour of SEPLA in clay. The model, named CASPA (Chain and SEPLA
Plasticity Analysis), was developed within classical plasticity theory and is able to predict forces and displacements during keying up to the peak load. A chain solution [8] was incorporated to account for the relationship between the chain loads and angles at both the padeye and at the mudline.

The performance of the CASPA model was assessed through comparison with Large Deformation Finite Element (LDFE) results and centrifuge experiments [7]. Using a rigid plasticity theory with associated flow rule, CASPA can well capture the ultimate resistance of the anchor, the rotation and loss of embedment up in the first stage of the keying process and the influence of different padeye offsets. However, the simplified nature of the employed macro-element model, especially in relation to rigid plasticity and the associated flow rule, resulted in incorrect predictions of the anchor trajectory including excessive initial backward movements and downward trajectories (i.e. re-embedment of the anchor) in the medium large displacement domain, for many padeye offset values. The concept of employing an associated flow rule to determine displacements and deformations has been applied to several geotechnical applications, yet all for relatively small deformations [7]. Conversely, anchor pull-out is a problem that involves large deformations.

This paper presents an improved macro-element model for plate anchors with (i) a non-associated plastic potential and (ii) based on rigid-plastic strain-hardening rule. The first additional ingredient aims at improving the prediction of the anchor trajectory for the whole displacement domain and for a large range of padeye offsets, while the second ingredient aims at providing prediction of force and displacement mobilisation at the early stages of the keying process. The performance of the

\footnotetext{
* Corresponding author: a.peccindasilva@bristol.ac.uk
} 
new model is assessed through comparison with previously published LDFE analyses and centrifuge tests [7, 9-11].

\section{Notation}

The notation adopted in this study is presented in Fig. 1. During the keying process, the pulling force $T_{a}$ is applied to the padeye, whose location is defined by the eccentricity $e_{n}$ normal to the fluke and the offset $e_{p}$ parallel to the fluke. The inclination of the pulling force at the padeye, which is nearly $90^{\circ}$ (vertical) immediately after installation, is identified by $\theta_{a}$. The inclination of the chain at mud-line level is indicated by $\theta_{0}$.

During the loading process, the anchor fluke is subjected to normal $(V)$, sliding $(H)$ and rotational $(M)$ forces, as well as the submerged anchor weight $W$, resulting in relative movements $\delta w, \delta u$ and $\delta \beta$ in the direction of $V, H$ and $M$, respectively. During the analysis, these relative movements are converted to the global coordinate system where $x$ and $z$ represent the horizontal and vertical displacements of the centre of the anchor, respectively.

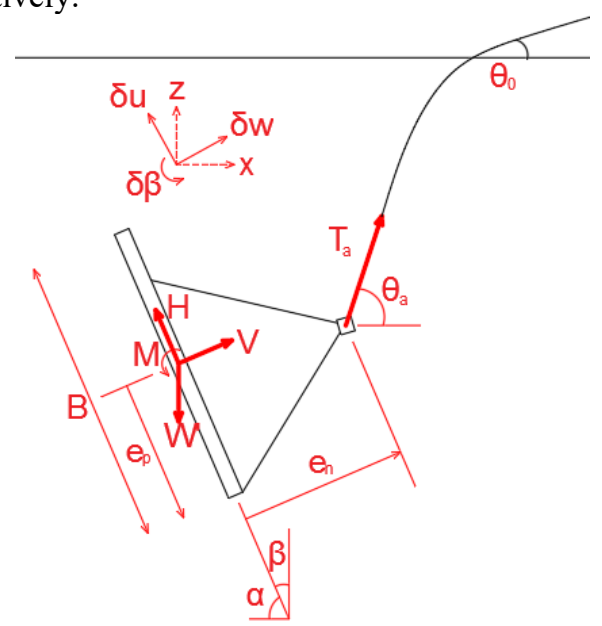

Fig. 1. Forces, displacements, rotations and eccentricities (adapted from [8])

\section{Description of the model}

Similar to CASPA [7], the newly proposed anchor model combines a macro-element module for the prediction of anchor behaviour with the chain solution proposed by Neubecker and Randolph [8]. A schematic 2-D overview of the proposed macro-element model is provided in Figure 2 in the normalised $H / H_{M}-V / V_{M}$ force plane $\left(M / M_{M}=0\right.$ for simplicity) where $V_{M}, M_{M}$ and $H_{M}$ are normal, rotational and sliding capacities when acting independently on the anchor. The model requires the definition of a loading surface, a strength surface and a plastic potential.

Loading and strength surfaces. The loading and the strength surfaces have the same form and follow the shape proposed by Cassidy et al. [7] and employed previously for drag anchors [12-13] and for plate anchors [14-15]. In the $V-H-M$ space (vertical, horizontal and moment loads), the strength surface $(F)$ is defined by Eq. 1.

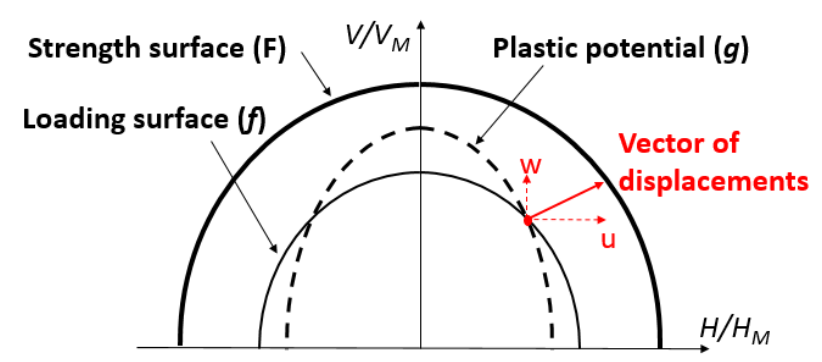

Fig. 2. Schematic 2-D view $\left(M / M_{M}=0\right)$ of the proposed model

$$
F=\left(\frac{V}{V_{M}}\right)^{q}-1+\left[\left(\frac{|M|}{M_{M}}\right)^{m}+\left(\frac{|H|}{H_{M}}\right)^{n}\right]^{1 / p}=0
$$

where $m, n, p, q$ are exponents that define the shape of the three-dimensional surface in the $V-H-M$ space. The capacities are usually represented by the factors $N_{v}, N_{h}$ and $N_{m}$, defined in Eq. 2, 3 and 4, respectively.

$$
\begin{aligned}
& N_{v}=V_{M} /\left(\begin{array}{lll}
L B & s_{u}
\end{array}\right) \\
& N_{h}=H_{M} /\left(\begin{array}{lll}
L & B & s_{u}
\end{array}\right) \\
& N_{m}=M_{M} /\left(\begin{array}{lll}
L & B^{2} & s_{u}
\end{array}\right)
\end{aligned}
$$

where $B$ and $L$ are the height and the width of the anchor and $s_{u}$ is the undrained shear strength of the soil. Several values for the parameters $N_{v}, N_{h}$ and $N_{m}$ were derived in previous studies. A value of 14 for $N_{v}$ was assumed from finite-element analysis for rectangular anchors [16]. The same value was used by Cassidy et al. [7]. For the moment capacity $N_{m}$, a value of 1.73 was yielded from finiteelement solutions [17]. However, since these simulations neglect the effect of the shank, a value of 2 has been adopted herein [7]. The value of the sliding parameter $N_{h}$ depends on the detailed anchor geometry and also on the material surface roughness. Cassidy et al. [7] reported a range of values varying between 2.78 and 3.41 and adopted a value of 3 , which was also used in the present study.

The loading surface $(f)$ is always smaller but has the same shape of the strength surface $(F)$ and is defined as:

$$
f=\left(\frac{V}{V_{M}}\right)^{q}-\rho_{c}+\left[\left(\frac{|M|}{M_{M}}\right)^{m}+\left(\frac{|H|}{H_{M}}\right)^{n}\right]^{1 / p}=0
$$

where $\rho_{c}$ is the strain hardening parameter defining the size of the loading surface and varying between 0 and 1 . When $\rho_{c}=1$, the loading surface coincides with the strength surface.

Strain-hardening rule. The strain-hardening term $\rho_{c}$ in Eq. 6 follows the proposal by Nova \& Montrasio [18] for shallow foundations:

$$
\rho_{c}=1-\exp \left\{-R_{0}\left[w^{2}+u^{2}+(B \beta)^{2}\right]^{1 / 2}\right\}
$$

where $R_{0}$ is the non-dimensional hardening parameter. The effect of the strain-hardening rule in the performance of the macroelement model will be further explored in this paper. 
Plastic potential and flow rule. Following the proposal by Nova and Montrasio [18], it is assumed that the plastic potential has a similar shape to the strength surface but with different intersections with the axis $V / V_{M}, H / H_{M}$ and $M / M_{M}$. This change in the intersections is achieved through the inclusion of the parameters $\gamma, \chi$ and $\psi$, which scale the normal, sliding and rotational capacities $\left(V_{M}, H_{M}\right.$ and $M_{M}$, respectively). As such, the equation of the plastic potential for the anchors has the following form:

$$
g=\left(\frac{V}{V_{M} / \gamma}\right)^{q}-\rho_{\mathrm{g}}+\left[\left(\frac{|M|}{M_{M} / \psi}\right)^{m}+\left(\frac{|H|}{H_{M} / \chi}\right)^{n}\right]^{1 / p}=0
$$

where parameter $\rho_{g}$ is a scaling factor whose numerical value has no practical relevance since only the derivatives of $g$ are of interest for the determination of the vector of anchor incremental displacements $\boldsymbol{\delta} \boldsymbol{q}(\delta w, \delta u$ and $\delta \beta)$. These quantities can be determined as follows:

$$
\boldsymbol{\delta} \boldsymbol{q}=\left(\begin{array}{c}
\delta w \\
\delta u \\
B \delta \beta
\end{array}\right)=\lambda\left(\begin{array}{c}
\partial g / \partial V \\
\partial g / \partial H \\
\partial g / \partial(M / B)
\end{array}\right)
$$

The term $\lambda$ is the plastic multiplier which can be determined by substituting Eq. 6 and 8 into the expression of the consistency condition shown in Eq. 9.

$$
d f\left(\boldsymbol{Q}, \rho_{c}\right)=\frac{\partial f}{\partial \boldsymbol{Q}} d \boldsymbol{Q}+\frac{\partial f}{\partial \rho_{c}} d \rho_{c}=0
$$

where $\boldsymbol{Q}=[V, H, M]^{\mathrm{T}}$.

$$
\lambda=\frac{-\left(\frac{\partial f}{\partial V} \dot{V}+\frac{\partial f}{\partial H} \dot{H}+\frac{\partial f}{\partial(M / B)} \dot{M}\right)}{\frac{\partial f}{\partial \rho_{C}}\left(\frac{\partial \rho_{C}}{\partial w} \frac{\partial g}{\partial V}+\frac{\partial \rho_{C}}{\partial u} \frac{\partial g}{\partial H}+\frac{\partial \rho_{C}}{\partial(B \beta)} \frac{\partial g}{\partial(M / B)}\right)}
$$

Force-equilibrium equations. While the macro-element model for the anchor provides the values of the normal, tangential and moment load at its centre, the force $T_{a}$ imposed by the chain at the padeye and its orientation $\left(\theta_{a}\right)$ is of great interest for practical purposes. By imposing force-equilibrium conditions in both translation directions and rotation of the anchor, the following relationships can be obtained:

$$
\begin{gathered}
V=T_{a} \sin \left(\beta+\pi / 2-\theta_{a}\right)-W^{\prime} \sin \beta \\
H=T_{a} \cos \left(\beta+\pi / 2-\theta_{a}\right)-W^{\prime} \cos \beta \\
M=T_{a}\left[e_{n} \cos \left(\beta+\pi / 2-\theta_{a}\right)+\right. \\
\left.e_{p} \sin \left(\beta+\pi / 2-\theta_{a}\right)\right]
\end{gathered}
$$

Chain solution. A chain load solution [8] expressed in Eq. 14 is included to relate the angle of pull at the mudline $\left(\theta_{0}\right)$ to the chain angle at the padeye $\left(\theta_{a}\right)$.

$$
\begin{array}{r}
e^{\mu\left(\theta_{a}-\theta_{0}\right)}\left(\cos \theta_{0}+\pi \sin \theta_{a}\right)-\cos \theta_{a}-\pi \sin \theta_{a}= \\
E_{n} d_{b a r} N_{c}\left(s_{u 0} z_{p}+\frac{k_{s u} z_{p}^{2}}{2}\right)\left(\frac{1+\mu^{2}}{T_{a}}\right)
\end{array}
$$

where $d_{b a r}$ is the diameter of the chain; $E_{n}$ is a multiplier giving the effective chain width in the normal direction to the chain; $N_{c}$ is the bearing capacity factor for the chain; $z_{p}$ is the current vertical depth of the padeye; $s_{u 0}$ and $k_{s u}$ are the soil shear strength at the mudline and the rate of increase with depth; and $\mu$ is the friction of the chain.

\section{Model calibration}

Calibration of 11 parameters is required for deriving the final form of the model: 4 shape parameters that define the loading surface $(m, n, p, q), 3$ normalised capacities $\left(N_{v}, N_{h}\right.$ and $\left.N_{m}\right)$ that determine the intersection of the loading surface with the axis, 3 parameters that define the size of the plastic potential surface $(\gamma, \chi$ and $\psi)$ and 1 hardening parameter $\left(R_{0}\right)$.

The three capacity factors $N_{v}, N_{h}$ and $N_{m}$ can be obtained from previous studies [7, 16, 17], as mentioned in the description of the model above. The values of the shape parameters $m, n, p$ and $q$ are also selected according to typical values found in the literature.

The parameter $p$ was reported to range from 1.01 to 1.10 for strip [12-13], rectangular [7] and square anchors [17]. Based on these previous studies, $p=1.0$ is adopted in this paper. The parameter $q$ was found to be close to 4.0 for square [17] and rectangular [7] anchors. Therefore, it is assumed herein that $q=4.0$. Parameter $n$ was reported to vary between 3.72 (square anchors, [17]) and 5.31 (strip anchors, [12-13]. Also, it was found to be equal 4.2 for rectangular anchors [7]. For the sake of simplicity, an intermediate value is selected $(n=4)$. Parameter $m$ was shown by the same authors to vary between 1.07 and 2.58 . For rectangular anchors, Cassidy et al. [7] used $m=1.1$. However, in this paper the value of $m=2$ is selected in order to avoid the existence of non-differentiable points in the loading surface. The parameters for the plastic potential $(\gamma, \chi, \psi)$ and the hardening parameter $\left(R_{0}\right)$ are calibrated with existing results from LDFE analyses carried out by Tian et al. [9]. Seven analyses (for seven padeye offsets $e_{p}$ ranging from 0 to $0.5 \mathrm{~B}$ ) were carried out in ABAQUS using the remeshing and interpolation technique with small strain (RITSS) [19], but an intermediate value of $e_{p} / B=0.3$ is selected for the calibration process. A square anchor with $B=L=5 \mathrm{~m}$ and thickness $t=0.25 \mathrm{~m}$ is simulated, with padeye eccentricity $e_{n}$ taken as $2.5 \mathrm{~m}\left(e_{n} / B=0.5\right)$. The soil profile is characterised by a linear strength increasing with depth, with $s_{u 0}=1.5 \mathrm{kPa}$ and $k_{s u}=1.5 \mathrm{kPa} / \mathrm{m}$. The parameters that showed the best fit are $\gamma=1.6, \chi=1.1, \psi=1.5$ and $R_{0}$ $=1.0$, and calibration against results from LDFE analysis is presented in Fig. 3. The parameters derived from the calibration process are presented in Table 1.

\section{Simulations}

To assess the performance of the macroelement model, additional simulations are performed and compared with previous analyses for other padeye offsets [9]. The results shown in Fig. 4 are in good agreement with LDFE simulations. The values of initial and final rotations of the anchor are well-captured by the model for all padeye offsets $\left(e_{p}\right)$ (Fig. 4a). Good agreement is also observed for the anchor trajectories, as displayed in Fig. 4b. Both horizontal and vertical movements are well-reproduced 
by the macro-element model with $\gamma=1.6, \chi=1.1, \psi=1.5$ and $R_{0}=1.0$. However, the horizontal displacements in the negative $x$ direction are slightly higher than the ones predicted by LDFE analyses; the loss of embedment $(z / B)$ is also slightly overestimated.
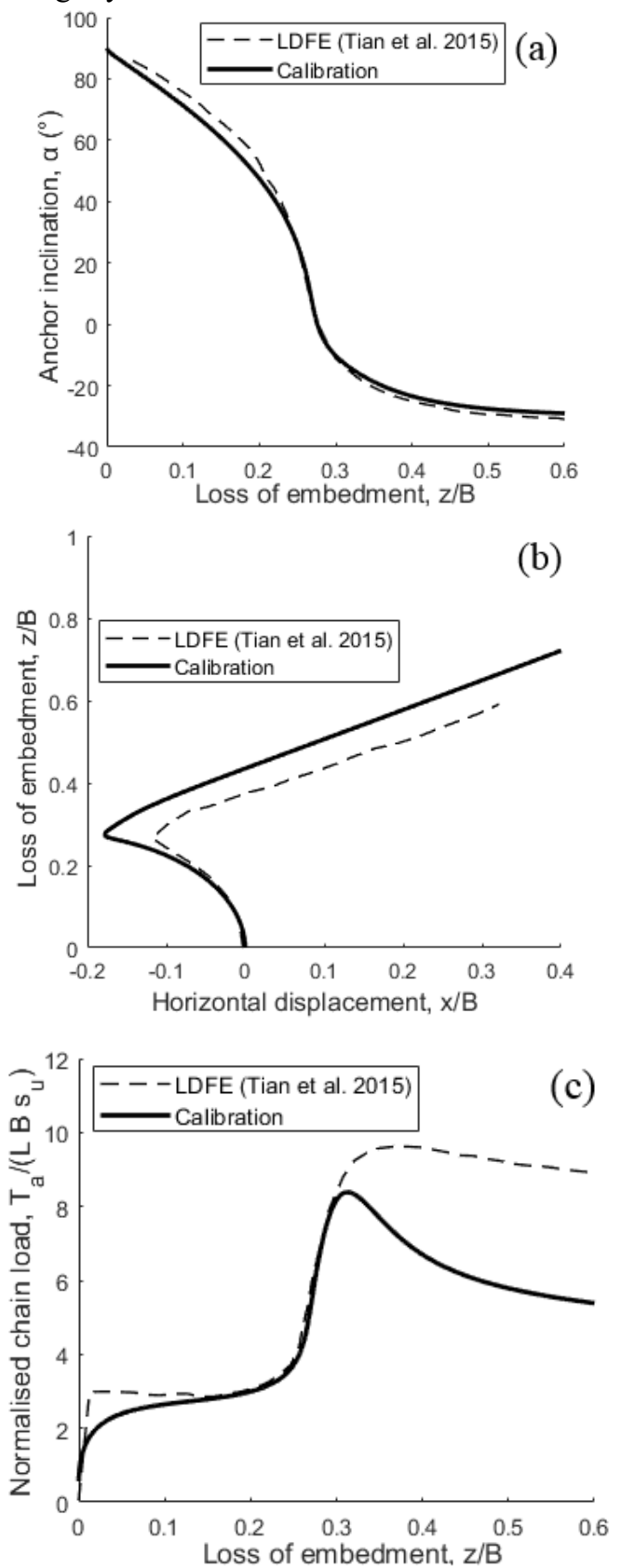

Fig. 3. Calibration of the new macro-element model: (a) anchor inclination, (b) anchor trajectory and (c) force-displacement.

Table 1. Summary of model parameters.

\begin{tabular}{c|l|c}
\hline Symbol & \multicolumn{1}{|c|}{ Description } & Value \\
\hline$m$ & Exponent (moment) & 2 \\
$n$ & Exponent (horizontal) & 4 \\
$p$ & Exponent (moment/horizontal) & 1 \\
$q$ & Exponent (vertical) & 4 \\
\hline$N_{v}$ & Normalised normal capacity & 14 \\
$N_{h}$ & Normalised sliding capacity & 3 \\
$N_{m}$ & Normalised moment capacity & 2 \\
\hline$\gamma$ & Plastic potential parameter (vertical) & 1.6 \\
$\chi$ & Plastic potential parameter (horizontal) & 1.1 \\
$\psi$ & Plastic potential parameter (moment) & 1.5 \\
\hline$R_{0}$ & Hardening parameter & 1.0
\end{tabular}

In terms of force-displacement (Fig. 4c), the macroelement model shows good prediction of the initial behaviour and the effect of padeye offset is well-captured. However, there seems to be an underestimation of the peak load - varying from 5.8 to $15.7 \%$. This divergence is believed to be related to the high dependence of anchor capacity prediction on the factors $N_{v}, N_{h}$ and $N_{m}$, which have been estimated from previous studies. In order to establish these factors accurately, the detailed geometry of the anchor must be considered, possibly in threedimensional FE analysis [7].
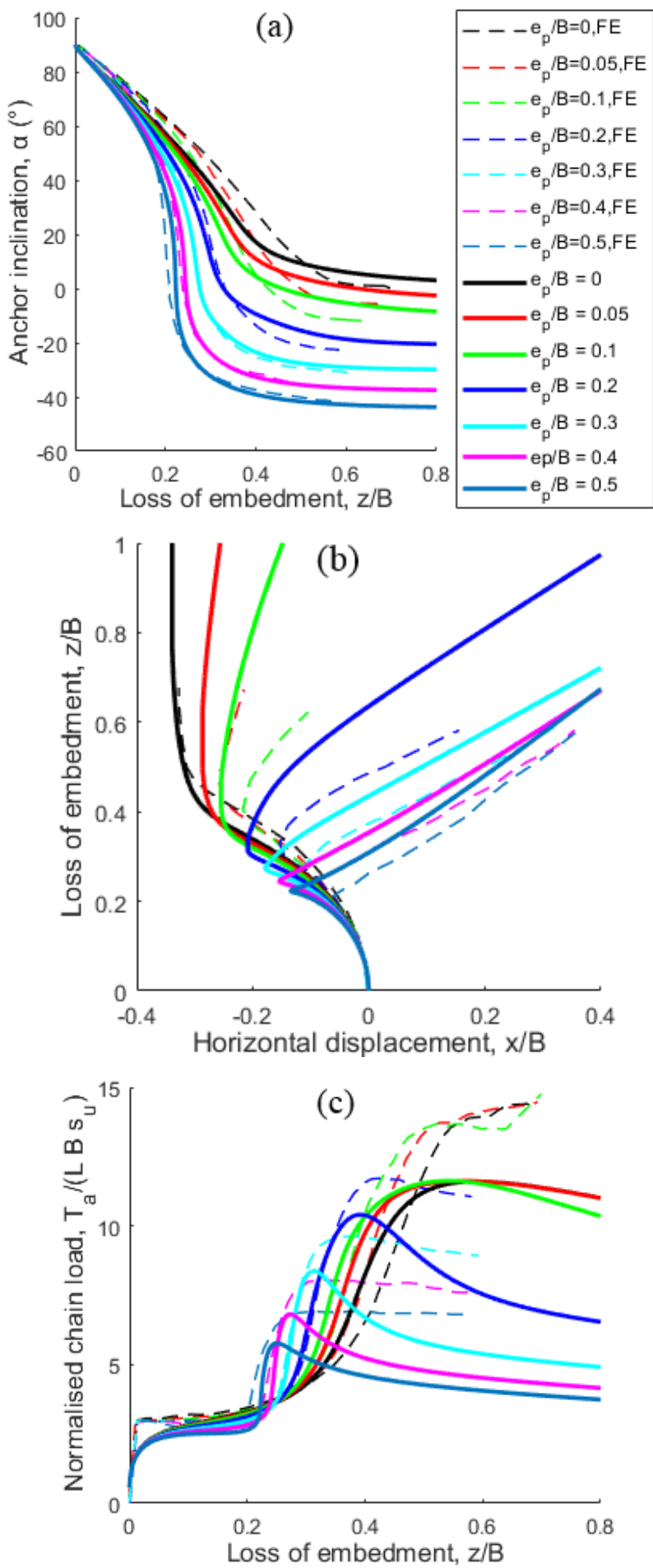

Fig. 4. Comparison of (a) anchor rotation, (b) anchor trajectory and (c) force-displacement of macro-element model with LDFE simulations [9] for $\mathrm{e}_{\mathrm{n}} / \mathrm{B}=0.5$. 
The performance of the new macro-element model is also verified for inclined chain load (with $\theta_{0}=60^{\circ}$ and $\theta_{a}$ calculated from the chain solution presented in Eq. 14), through comparison with LDFE analyses [10] and with centrifuge tests [11]. The experimental set-up for the centrifuge test [11] consisted of a square anchor with equivalent prototype dimensions of $4 \mathrm{~m} \mathrm{x} 4 \mathrm{~m} \times 0.2 \mathrm{~m}$, eccentricity ratio $e_{n} / B=0.625$ and a transparent soil with uniform strength profile $\left(s_{u}=18 \mathrm{kPa}\right)$. The anchor centroid was installed at an initial depth of three times the anchor height. LDFE analyses [10] were conducted in the FE software AFENA, with the implementation of the RITSS approach [19]. The anchor was modelled with and without the shank.

These results are presented in Fig. 5 and show that the inclusion of a non-associated plastic potential and a strainhardening rule significantly improves the prediction of anchor inclination in comparison with the model with associated plastic flow and no hardening rule. The rotational behaviour of the anchor predicted by the new model shows similar results to centrifuge tests and LDFE analysis with the shank up to the stage where the anchor has rotated to an inclination of $40^{\circ}$ to the horizontal. Beyond that point both centrifuge test and LDFE analyses show that the rotation tends to stabilise at an angle of about $37^{\circ}$. The new model, on the other hand, predicts that the anchor continues to rotate up to an angle of about $26^{\circ}$.

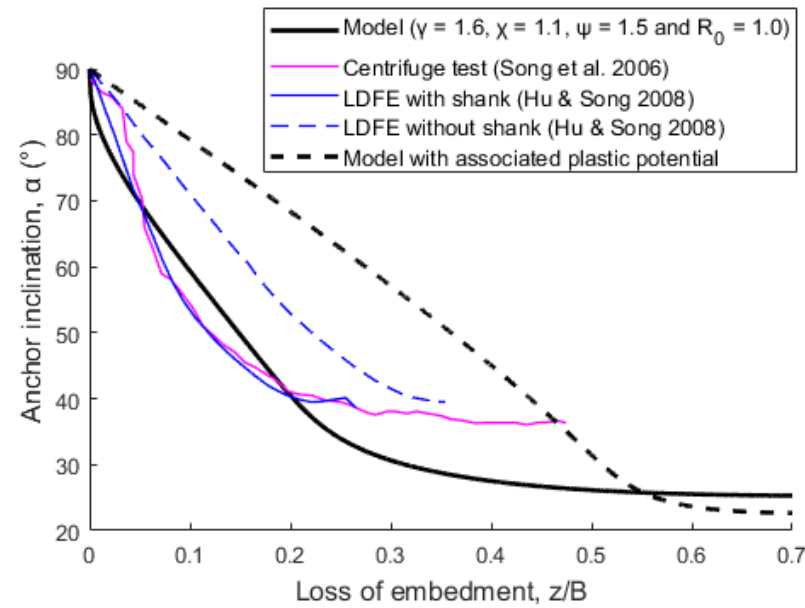

Fig. 5. Comparison of anchor rotation obtained from centrifuge tests [11], LDFE analyses [10] and macroelement models.

The effect of the inclusion of the new features (nonassociated plastic potential and strain-hardening rule) can be visualised in Fig. 6, which shows the difference between the vectors of displacement and the strength surface. With the inclusion of a non-associated plastic potential surface, the direction of the displacement increments becomes dependent on this new surface, although the loading path is not affected. The first important remark is that with the inclusion of a strainhardening rule, the loading path starts from inside the surface as opposed to the purely plastic model in which the loading path always lies on the strength surface. The second clear remark is that the vectors of increments of displacement are no longer normal to the strength surface represented in grey as in the model with associated plastic potential.

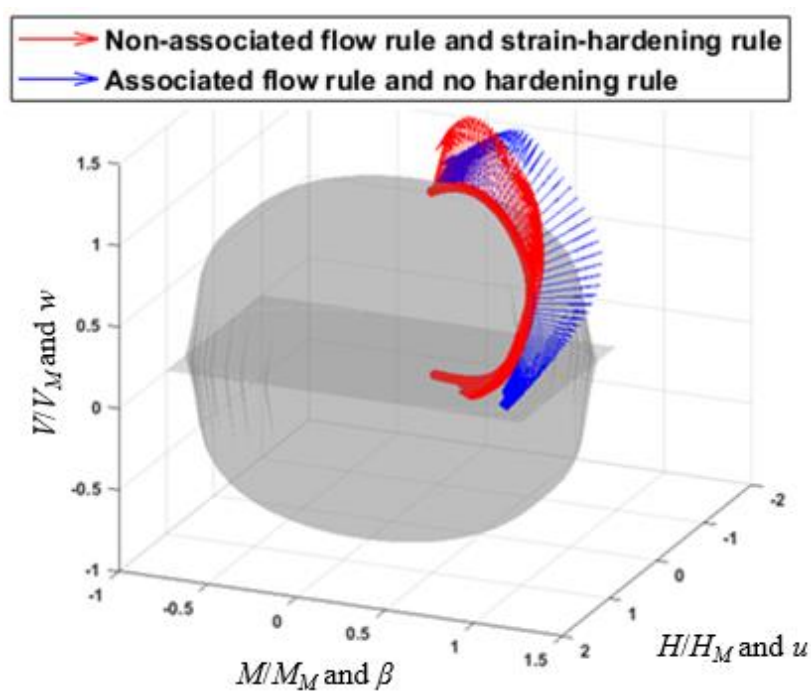

Fig. 6. Loading paths and vectors of displacement increments

The performance of the new macro-element model is also verified through comparison with the results published by Cassidy et al. [7], as shown in Fig. 7. The anchor trajectory (Fig. 7a) predicted by the model with non-associated plastic potential and strain-hardening rule shows good agreement with LDFE results and centrifuge PIV. The result obtained with the parameters selected from the calibration process $(\gamma=1.6, \chi=1.1, \psi=1.5$ and $\left.R_{0}=1.0\right)$ are similar to the best fit $(\gamma=1.5, \chi=1.1, \psi=$ 1.55 and $\left.R_{0}=1.0\right)$. The movement in the negative $x$ direction is reduced in comparison with the model with associated plastic potential. In the new model, the anchor moves upwards and does not embed again as in the model with associated plastic potential. The deviation with respect to the centrifuge PIV beyond $x / B=0.2$ can be attributed to the activation of the keying flap [7] which is not explicitly accounted for in LDFE and macro-element simulations.

The reason for the anchor to embed again beyond a certain point in the model with associated plastic potential can be attributed to the shape of the yield envelope [7], which results in a greater tendency for the anchor to fail in a sliding mode. The inclusion of a non-associated plastic potential reduces the displacement increments in the sliding (horizontal) direction and increases the normal (vertical) and rotational (moment) displacements, causing more rotation and movements in the normal direction and less sliding movement.

Anchor rotation (Fig. 7b) predicted by the new model also shows good agreement with FE results and reasonably good agreement with centrifuge PIV. The model with non-associated flow rule and strain-hardening rule shows that the anchor continues to rise vertically, as opposed to the model with associated plastic potential which showed that beyond a certain point $\left(\alpha \approx 40^{\circ}\right)$ the anchor starts to embed again. The deviation between the centrifuge PIV and LDFE results can be attributed to the experimental set-up, where the anchor is continuously pulled (with $\theta_{0}$ increasing progressively and the chain cutting through the soil) and not dragged with $\theta_{0}$ remaining constant, as implied by LDFE models and for the new model. 

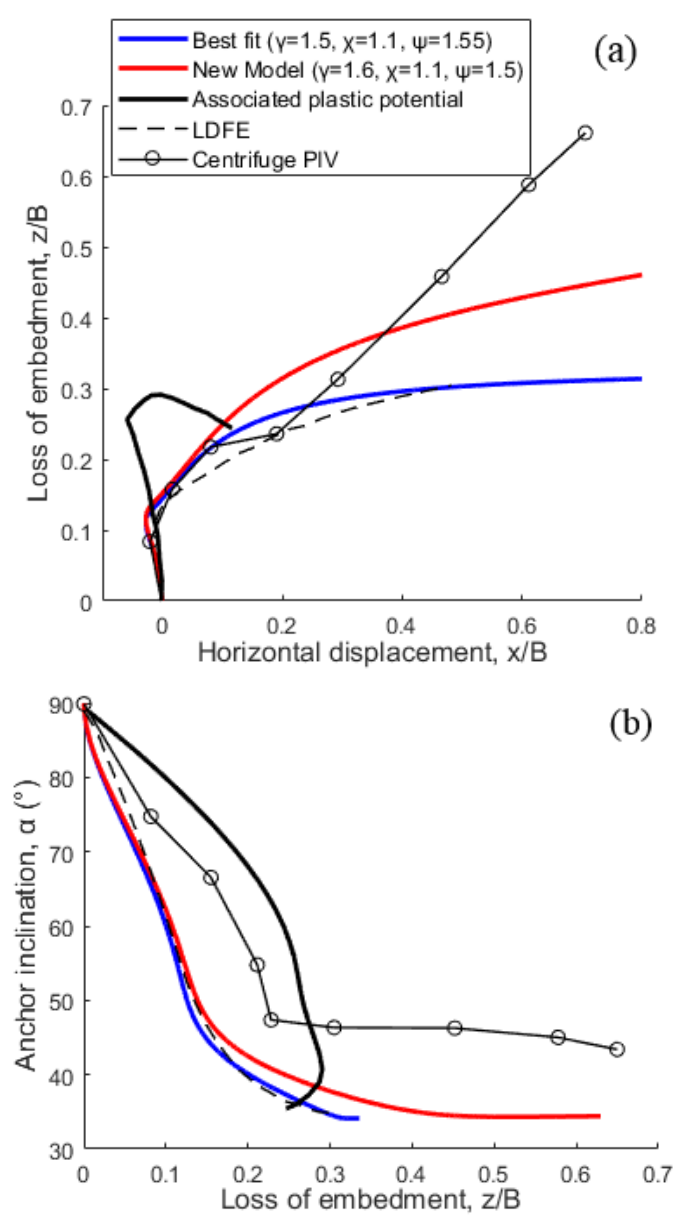

Fig. 7. Comparison of (a) anchor rotation and loss of embedment; and (b) trajectory, for centrifuge test [7], LDFE [7] analysis and macro-element models.

\section{Concluding remarks}

A new macro-element model was proposed in this paper and compared to a previously published model. The proposed new model includes: (i) a non-associated plastic potential and (ii) a strain-hardening rule into a rigidplasticity theory framework. Four new parameters (three for the plastic potential and one for the hardening rule) were included and calibrated with a LDFE analysis [9] and validated through comparison with three other studies [7, 10, 11], including LDFE simulations and centrifuge PIV tests. The inclusion of the new features was found to be successful in improving simulations of anchor trajectory and rotation during both the keying and loading process.

\section{References}

[1] P. Dove, H. Treu, B. Wilde. Suction embedded plate anchor (SEPLA): A new anchoring solution for ultradeepwater mooring. Proc. Deep Offshore Tech. Conf., New Orleans (1998).

[2] B. Wilde, H. Treu, T. Fulton. Field testing of suction embedded plate anchors. Proc. 11th Int. Offshore and Polar Eng. Conf., Stavanger, 544-551 (2001).

[3] C. Gaudin, Y. Tian, M.J. Cassidy, M.F. Randolph, C. O'Loughlin. Design and performance of suction embedded plate anchors. Proc. 3rd Int. Symp. Frontiers in Offshore Geotech, Oslo, Norway. CRC Press, Leiden, the Netherlands, pp. 863-868 (2015).

[4] Z. Song, Y. Hu, C. O’Loughlin, M. Randolph. Loss in anchor embedment during plate anchor keying in clay. $J$. Geotech. Geoenviron. Engng, 135, No. 10, 1475-1485 (2009).

[5] M. Yang, C.P. Aubeny, J. D. Murff. Behavior of suction embedded plate anchors during keying process. $J$. Geotech. Geoenviron. Engng 138, No. 2, 174-183 (2012). [6] F. Pisano, L. Flessati, C. di Prisco. A macroelement framework for shallow foundations including changes in configuration. Géotechnique, 62, No. 11, 910-926 (2016). [7] M. J. Cassidy, C. Gaudin, M.F. Randolph, P.C Wong, D. Wang, Y. Tian. A plasticity model to assess the keying of plate anchors. Géotechnique, 62, No. 9, 825-836 (2012).

[8] S.R. Neubecker, M.F. Randolph. Profile and frictional capacity of embedded anchor chains. J. Geotech. Engng, 121, No. 11, 797-803 (1995).

[9] Y. Tian, C. Gaudin, M.F. Randolph, M.J. Cassidy. Influence of padeye offset on bearing capacity of threedimensional plate anchors. Can. Geotech. J. 52, No. 6, 682-693 (2015).

[10] Y. Hu, Z. Song. Large deformation FE Analysis of plate anchor keying in clay. Proc. $12^{\text {th }}$ Int. Conf. of IACMAG, Goa, India, pp. 3299-3306 (2008).

[11] Z. Song, Y. Hu, D. Wang, C.D. O'Loughlin. Pullout capacity and rotational behaviour of square anchors in kaolin clay and transparent soil. Proc. Int. Conf. on Physical Modelling in Geotechnics, Hong Kong, China, 4-6 August, 1325-1331 (2006).

[12] M.F. Bransby, M. O’Neill. Drag anchor fluke soil interaction in clays. Proc. 7th Int. Symp. on Numerical Models in Geomechanics, Graz, 489-494 (1999).

[13] S. Elkhatib, M.F. Randolph. The effect of interface friction on the performance of drag-in plate anchors. Proc. 5th Int. Symp. on Frontiers in Offshore Geotech, Perth, $171-177$ (2005).

[14] J. D. Murff, M.F. Randolph, S. Elkhatib, H.J. Kolk, R. Ruinen, P.J. Strom, C. Thorne. Vertically loaded plate anchors for deep water applications. Proc. 5th Int. Symp. on Frontiers in Offshore Geotech, Perth, 31-48 (2005).

[15] M. Yang, C.P. Aubeny, J.D. Murff. Behavior of suction embedded plate anchors during keying process. $J$. Geotech. Geoenviron. Engng 138, No. 2, 174-183 (2012). [16] D. Wang, Y. Hu, M.F. Randolph. Keying of rectangular plate anchors in normally consolidated clay. J. Geotech. Geoenviron. Engng 137, No. 12, 1244-1253 (2011).

[17] S. Elkhatib. The behaviour of drag-in plate anchors in soft cohesive soils. $\mathrm{PhD}$ thesis, The University of Western Australia (2006).

[18] R. Nova, L. Montrasio. Settlements of shallow foundations on sand. Géotechnique, 41, No. 2, 243-256 (1991).

[19] Y. Hu, M.F. Randolph. A practical numerical approach for large deformation problems in soil. Int. J. Numer. Anal. Methods Geomech., 22, No. 5, 327-350 (1998). 